\title{
The Only Constant is Change: New ICD-10 Codes in Neonatology
}

Scott D. Duncan, MD, MHA

"The philosopher Heraclitus stated, "the only constant in life Is change." With nearly 30 years of experience as a neonatologist and 45 years within the hospital setting, Heraclitus' adage holds true when considering healthcare. Advances in knowledge surrounding technology, genetics, diagnostic evaluations, and therapeutic interventions remain at the forefront in the practice of most clinicians."

The philosopher Heraclitus stated, "the only constant in life Is change." With nearly 30 years of experience as a neonatologist and 45 years within the hospital setting, Heraclitus' adage holds true when considering healthcare. Advances in knowledge surrounding technology, genetics, diagnostic evaluations, and therapeutic interventions remain at the forefront in the practice of most clinicians. Strategies employed to "keep current" include reviewing recent literature, attending conferences, workshops, CME events, MOC activities, etc. However, physicians often ignore the changing facets of the business of neonatology.

Both Current Procedural Terminology $\left(\mathrm{CPT}^{\circledR}\right)$ and International Classification of Disease (ICD-10-CM) codes are updated on a regular timetable. The $\mathrm{CPT}^{\circledR}$ Editorial Panel) maintains the $\mathrm{CPT}^{\circledR}$ code set, supported by the $\mathrm{CPT}^{\circledR}$ Advisory Committee. CPT $^{\circledR}$ codes are updated annually, with their use begins on January 1 each year. (1) ICD-10 codes are also updated annually by the Centers for Medicare \& Medicaid Services (CMS). Announcements and updates for the 2022 ICD-10-CM, effective October 1, 2021 , can be found on the CMS website (2), as well as guidelines for coding and reporting for the current year. (3)

October 1 finds the expansion of two ICD-10-CM code sets of significance to neonatologists. (4)

The first is the addition of the P09 code set, which adds Abnormal Findings on Neonatal Screening. Included in this set are:

- P09.1 Abnormal findings on neonatal screening for inborn errors of metabolism

- $\quad$ P09.2 Abnormal findings on neonatal screening for congenital endocrine disease

- $\quad$ P09.3 Abnormal findings on neonatal screening for congenital hematologic disorders

- P09.4 Abnormal findings on neonatal screening for cystic fibrosis

- P09.5 Abnormal findings on neonatal screening for critical congenital heart disease
- P09.6 Abnormal findings on neonatal screening for neonatal hearing loss

- $\quad$ P09.8 Other abnormal findings on neonatal screening

- P09.9 Abnormal findings on neonatal screening, unspecified

The second expansion is of the P00.8 code set to include P00.82, Newborns affected by (positive) maternal group B streptococcus (GBS) colonization. A type 2 exclusion exists for P00.2 Newborn affected by maternal infectious and parasitic disease. If a different infectious and/or parasitic disease affects the newborn, both P00.82 and P00.2 may be reported.

$\mathrm{Q}$ : An infant is born via vaginal delivery to a GBS-positive mother following rupture of membranes for 26 hours. The maternal temperature peaked at $101^{\circ} \mathrm{F}$, and the mother received two doses of Penicillin greater than 4 hours prior to delivery. The infant is well appearing. Using the Neonatal Early-Onset Sepsis Calculator, the neonatologist orders vital signs every 4 hours for 24 hours. The correct ICD-10-CM code is:
A. P00.2
B. $\mathrm{P} 02.7$
C. $\mathrm{P} 00.82$
D. Z05.1

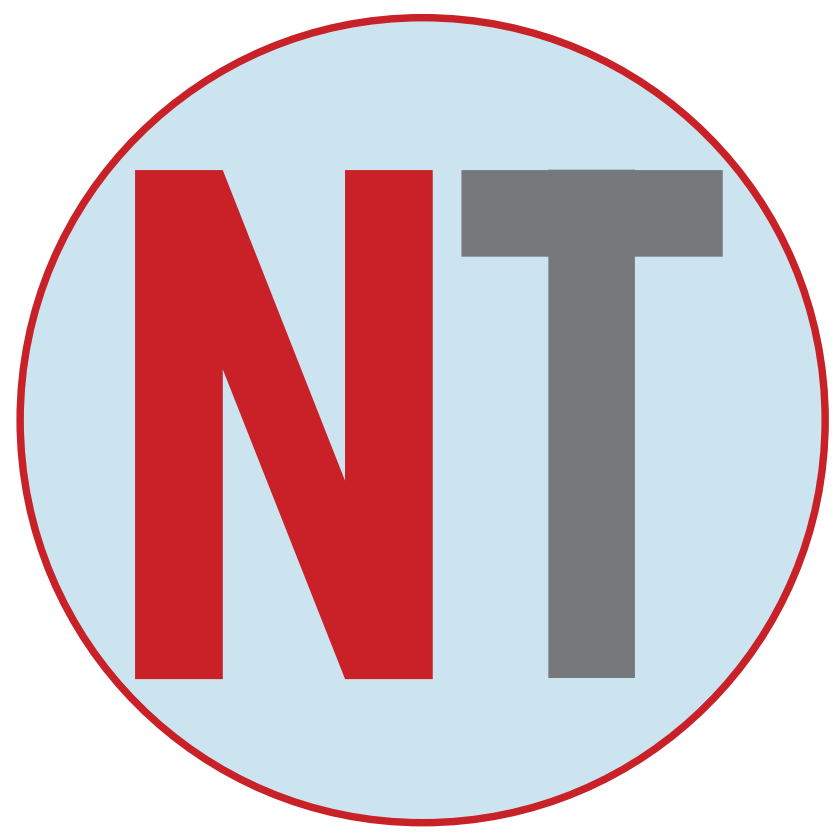

The correct answer is $B$. The evaluation was undertaken due to the maternal history of GBS colonization associated with the maternal and neonatal clinical presentation. P00.2 is incorrect as there are no other maternal infectious complications recorded. P02.7 is a Newborn affected by chorioamnionitis. This diagnosis would be applicable should the obstetrician determine that the mother had chorioamnionitis. Z05.1 Observation and evaluation of newborn for suspected infectious condition ruled out would be used once the evaluation was determined to be negative. 
Most neonatologists would rather concentrate on patient care; however, at least one member of the practice should focus on the business aspects of the practice. Areas of emphasis should be on proper CPT (charges) and ICD-10 (diagnosis) coding. The American Academy of Pediatrics provides numerous resources to allow the physician to stay up to date, including publications such as the Pediatric Coding Newsletter, Coding for Pediatrics, Pediatric ICD-10-CM, as well as AMA publications such as CPT Professional Edition and ICD-10-CM. Section members have access to the Coding Committee for questions and educational content. In addition, questions can be submitted to the Coding Corner via the web (email) or through the Coding Hotline (web) at https://form. jotform.com/Subspecialty/aapcodinghotline.

\section{References:}

1. American Medical Association. CPT Editorial Panel: The CPT code process. Accessed October 8, 2021, https://www. ama-assn.org/about/cpt-editorial-panel/cpt-code-process

2. Centers for Medicare \& Medicaid Services. 2022 ICD-10CM. Accessed October 8, 2021, https://www.cms.gov/medicare/icd-10/2022-icd-10-cm

3. Centers for Medicare \& Medicaid Services. ICD-10-CM Official Guidelines for Coding and Reporting; FY 2022. Accessed October 8, 2021, https://www.cms.gov/files/document/fy-2022-icd-10-cm-coding-guidelines.pdf

4. American Academy of Pediatrics. AAP Pediatric Coding Newsletter. 2021;16(12):4.

Disclosure: The author has no disclosures.

NT

Corresponding Author:

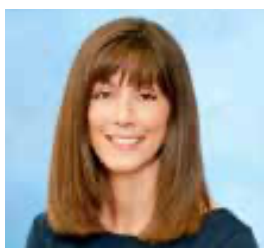

Kate Peterson Stanley, MD

Medical Director of Revenue Integrity

CS Mott Children's and Von Voigtlander Women's Hospitals

Clinical Assistant Professor

Division of Neonatal-Perinatal Medicine

Department of Pediatrics

University of Michigan Medical School

Mailing Address: 8-621 CS Mott

1540 E. Hospital Dr., SPC4254

Ann Arbor, MI 48109-4254

Phone: 734-763-4109

Fax: 734-763-7728

Email: katest@med.umich.edu

\section{SAFETY IN THE NICU}

\section{New tubes, new problems?}

A new tubing design meant to eliminate tubing misconnections has introduced new challenges for the NICU population. Pediatric providers must deliver medication in small volumes to tiny patients with high levels of accuracy. The new tubing design, known as ENFit ${ }^{\circledR}$, could present dosing accuracy and workflow challenges.
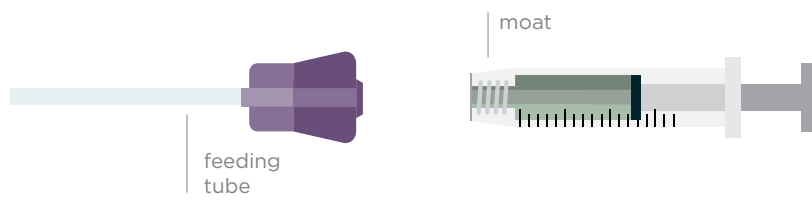

DOSING ACCURACY

- The moat, or area around the syringe barrel, is difficult to clear. Medication can hide there, inadvertently increasing the delivered dose when the syringe and feeding tube are connected; patients may receive extra medication.

INFECTION RISK

- The moat design can increase risk for infection if residual breast milk or formula remains in the moat and transfers to the feeding tube.

\section{WORKFLOW ISSUES}

- Increased nursing workflow is seen with additional steps for clearing syringe moats, cleaning tube hubs, and using multiple connectors.

Improved standards are important to protect patients from the dangers of tubing misconnections. But we must avoid mitigating existing risks by creating new ones.

Individual hospitals should consider all factors impacting their NICU patients before adopting a new tubing design.

ENFit ${ }^{\oplus}$ is a registered trademark of GEDSA

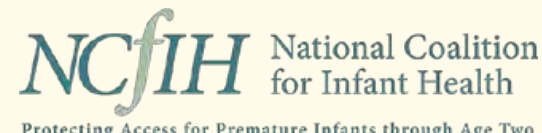

A collaborative of professional, clinical, community health, and family support organizations focused on the health and safety of premature infants.

infanthealth.org 